\title{
Drug treatment of type 2 diabetes mellitus in patients for whom metformin is contraindicated [Corrigendum]
}

\author{
Irons BK, Minze MG. Drug treatment of type 2 diabetes
} mellitus in patients for whom metformin is contraindicated. Diabetes Metab Syndr Obes. 2014;7:15-24.

There is an error in the text on page 19:

"Alpha-glucosidase inhibitors are infrequently used in the treatment of T2DM. ${ }^{3}$ These agents competitively inhibit the absorption of alpha-glucosidase along the brush border of the small intestine, which slows gut absorption of carbohydrates and ultimately reduces post-prandial blood glucose. ${ }^{34-36 "}$ should read as "Alpha-glucosidase inhibitors are infrequently used in the treatment of T2DM. ${ }^{3}$ These agents competitively inhibit the degradation of complex carbohydrates along the brush border of the small intestine, which slows gut absorption of metabolized carbohydrates and ultimately reduces post-prandial blood glucose. ${ }^{34-36 "}$ 